\title{
The effect of overtraining on the shift behavior of albino rats'
}

\author{
Thomas J. Tighe, Paul L. Brown and Edward A. Youngs \\ DARTMOUTH COLLEGE
}

\begin{abstract}
Rats were trained to criterion to respond to 1 dimension of a 2-dimensional discrimination and then to respond either (a) to the previously negative stimulus or (b) to the previously irrelevant dimension. One half of the Ss received 200 training trials beyond criterion before undergoing the discrimination shifts. Overtraining had no effect, and (b) was accomplished faster than (a) by both criterion and overtrained Ss.
\end{abstract}

\section{Problem.}

Adult humans learn a reversal shift (RS), which involves a reversal of response to a previously relevant dimension, more rapidly than an extradimensional shift (EDS), which requires response to a previously irrelevant dimension. On the other hand, infrahuman organisms and very young children have been found to accomplish an EDS more easily than an RS (Kendler \& Kendler, 1962). The present experiment is concerned with an attempt to determine the extent to which these age- and species-differences may be attributed to training variables common to the human and animal levels rather than to the operation of variables specific to human development. The possibility that special training may enable infrahuman Ss to learn an $\mathrm{RS}$ more rapidly than an EDS is suggested both by several studies on the relation between overtraining and discrimination reversal (e. g., Reid, 1953) and by theoretical treatments of the comparative data on shift behavior.

\section{Method}

Forty-eight male albino rats between 90 and 120 days old were trained in an apparatus patterned after Gibson \& Walk (1956). The stimuli were presented to $S$ at an angle of $25^{\circ}$ from the upright and varied simultaneously in two aspects-horizontal vs. vertical black stripes ( $1 / 4$ in wide) placed on flat vs. raised white $21 / 2$ in squares (i. e., a two-dimensional vs. a threedimensional representation). The flat squares were painted in the center of $43 / 8 \times 7$ in gray metal plaques. The raised squares were $3 / 4$ in thick metal blocks painted white and centered on similar plaques. Thus, the stimuli presented to S on any one trial (according to a prearranged counterbalanced order) consisted of one of the following two pairs: (a) flat square with vertical stripes vs. raised square with horizontal stripes, or (b) flat square with horizontal stripes vs. raised square with vertical stripes. Each square had a $11 / 8$ in square hinged door cut in its center. Behind the door was a small metal food tray which always contained a $94 \mathrm{mg}$ food pellet. A correct response consisted of nosing open the unlatched door of S+ to get the pellet, while an in- correct response consisted of nosing S- which was latched from behind.

Each S underwent pretraining during which he became accustomed to the apparatus and learned to open the doors and eat from the food trays. All Ss then learned a discrimination with one of the following as the S+: flat square $(F)$, raised square (R), horizontal stripes $(H)$, or vertical stripes (V). For example, if an $\mathrm{S}$ had $\mathrm{H}$ as $\mathrm{S}+$, he was reinforced for responding to horizontal stripes regardless of whether they appeared on the flat or on the raised square. Upon reaching criterion in the initial discrimination half of the Ss (Criterion group) were immediately given the discrimination shifts. Half of these Ss received a reversal shift (Group C-RS) and the other half received an extradimensional shift (Group C-EDS). For example, if an S had $\mathrm{H}$ as $\mathrm{S}+$ in the initial discrimination he could then be given either an $\mathrm{RS}$ to $\mathrm{V}$ as $\mathrm{S}+$ or an EDS to $\mathrm{F}$ or $\mathrm{R}$ as $\mathrm{S}+$. The remaining Ss, Group OT, were similarly treated but only after having received 200 additional training trials on the initial discrimination during which criterion performance had to be maintained. This figure represents about $200 \%$ of the mean trials required to complete the original learning. The Ss received 20 noncorrection trials per day in each phase of the experiment, and were run until a criterion of 18 of them, including 9 out of the last 10 , were correct, both in the original learning phase and for the post-shift discriminations. Overtraining was given on consecutive days until 10 days of criterion performance had been achieved. During the post-shift discriminations the irrelevant dimension was held constant in order to minimize possible partial reinforcement effects for Ss undergoing the EDS. Each stimulus dimension was used equally often as the relevant dimension during original learning and the discrimination shifts. Each discrimination was begun on the day following completion of the preceding task.

\section{Results and Discussion}

The major treatment groups did not differ in speed of learning the initial discrimination $(F<1)$. All but 5 of the Ss completed overtraining (OT) in 10 days. These 5 animals ( 2 in the RS group and 3 in the EDS group) each required 1 additional day. During OT the mean number of errors per day was .29. The shift performance of the animals is summarized in Table 1. Trial and error scores for the discrimination shifts are presented in terms of the dimension involved since the $\mathrm{H}$ vs. $\mathrm{V}$ discrimination was significantly more difficult than the $\mathbf{F}$ vs. $R$ discrimination. Factorial 
Table 1

Mean Number of Errors and Trials to Criterion during Shifts

(Each subgroup contains $6 \underline{\text { Ss}}$ )

Group

Relevant Dimension

\begin{tabular}{lrccc} 
& \multicolumn{2}{c}{ Horizontal-Vertical } & \multicolumn{2}{c}{ Flat-Raised } \\
& Errors & Trials & Errors & Trials \\
& & & & \\
C-RS & 111.0 & 196.7 & 42.7 & 86.7 \\
OT-RS & 96.0 & 170.0 & 56.3 & 90.0 \\
C-EDS & 56.0 & 126.7 & 21.0 & 66.7 \\
OT-EDS & 43.3 & 110.0 & 23.3 & 63.3
\end{tabular}

analyses of variance were applied to these trial and error scores transformed to natural logarithms. The Ss in the EDS groups required significantly fewer trials and errors to reach criterion than did Ss in the RS groups (F, 11.84; df, 1/40; $<$. .001; F, 35.46; df, 1/40; $\mathrm{p}<.001$; for the trial and error measures, respectively). Overtraining was not a significant source of variance ( $F<1$ for both measures). Both types of shift were accomplished significantly more rapidly when the $\mathbf{F}$ vs. $\mathbf{R}$ discrimination was relevant as opposed to the $\mathrm{H}$ vs. $\mathrm{V}$ discrimination (trials - F, 28.53; df, 1/40; $\mathrm{p}<.001)$. There were no significant interaction effects ( $F<1$ in each case). The mean number of errors before the first correct response following reversal shift was 22.0 for the overtrained group and 7.3 for the criterion group $(t=2.7, p<.02$, two tailed).

The finding that rats accomplish an EDS more rapidly than an RS confirms Kelleher's experiment with rats (1956), which employed different dimensions than those used here. The fact that OT had no effect on relative ease of shift is in agreement with the failure to find an effect of OT on the discrimination shift behavior of rhesus monkeys, who were also found to accomplish an EDS more rapidly than an RS (Tighe, 1964b). Although there is some evidence to suggest that the differences between the discrimination shift behavior of human and infrahuman Ss maybe eliminated by an OT procedure (e. g., Mackintosh, 1962), the present pattern of results taken together with recent failures to find the overlearning-reversal effect (e. g., Erlebacher, 1963) indicate that such a relation, if established, would be of a limited and conditional nature.

The ease with which the rats learned the $F$ vs. $R$ discrimination in the present experiment is also noteworthy. Their performance in this respect resembled that of rhesus monkeys in the discrimination of virtually identical stimuli in similar tasks (Tighe, 1964a), thus indicating that relatively high sensitivity to the differences between objects and patterns is common to species which differ widely in visual capacity.

\section{References}

ERLEBACHER, A. Reversal learning in rats as a function of percentage of reinforcement and degree of learning. J. exp. Psychol., 1963, 66, 84-90.

GIBSON, E. J., \& WALK, R. D. The effect of prolonged exposure to visually presented patterns on learning to discriminate them. J. comp. physiol. Psychol., 1956, 49, 239-242.

KELLEHER, R. I. Discrimination learning as a function of reversal and nonreversal shifts. J. exp. Psychol., 1956, 51, 379-384.

KENDLER, H. H., \& KENDLER, T. S. Vertical and horizontal processes in problem solving. Psychol. Rev., 1962, 69, 1-16.

MACKINTOSH, N. J. The effects of overtraining on a reversal and a nonreversal shift. J. comp. physiol. Psychol., 1962, 55, 555-559.

REID, L. S. The development of noncontinuity behavior through continuity learning. J. exp. Psychol., 1953, 46, 107-112.

TIGHE, T. J. Reversal and nonreversal shifts in monkeys. J. comp. physiol. Psychol., 1964a, 58, 324-326.

TIGHE, T. J. The effect of overtraining on reversal and nonreversal shifts in monkeys. Paper read at Eastern Psychol. Ass., Philadelphia, April, 1964b.

Note

1. This research was supported by grant GB 1673 from the National Science Foundation to the senior author. 